\title{
Étude qualitative et quantitative des peuplements phyto- et zooplanctoniques dans les bassins de la saline de Sfax, Tunisie Qualitative and quantitative study of phyto- and zooplankton communities in the saline pounds of Sfax, Tunisia
}

\author{
H. Ayadi, N. Toumi, O. Abid, K. Medhioub, M. Hammami, T. Sime-Ngando, C. \\ Amblard et D. Sargos
}

Volume 15, numéro 1, 2002

URI : https://id.erudit.org/iderudit/705441ar

DOI : https://doi.org/10.7202/705441ar

Aller au sommaire du numéro

\section{Éditeur(s)}

Université du Québec - INRS-Eau, Terre et Environnement (INRS-ETE)

ISSN

0992-7158 (imprimé)

1718-8598 (numérique)

Découvrir la revue

Citer cet article

Ayadi, H., Toumi, N., Abid, O., Medhioub, K., Hammami, M., Sime-Ngando, T., Amblard, C. \& Sargos, D. (2002). Étude qualitative et quantitative des peuplements phyto- et zooplanctoniques dans les bassins de la saline de Sfax, Tunisie. Revue des sciences de l'eau / Journal of Water Science, 15(1), 123-135. https://doi.org/10.7202/705441ar
Résumé de l'article

Le plancton végétal et animal a été analysé dans différents bassins de la saline de Sfax (Tunisie), entre le mois d'août 1999 et le mois de mai 2000. Des communautés diversifiées de phyto- et de zooplancton ont été observées. Quelle que soit la saison considérée, la structure des peuplements phytoplanctoniques se caractérise par la dominance de diatomées dans les bassins primaires de la saline (salinité $<200 \%$ ), suivie d'une dominance de dinoflagellés dans les bassins intermédiaires (salinité 200-300 \%), et d'un développement quasi-monospécifique de la chlorophycée Dunaliella salina dans les bassins terminaux où cristallise et se dépose la production saline (salinité > $300 \%$ ). En ce qui concerne le zooplancton (> $20 \mu \mathrm{m}$ ), les crustacés (copépodes) et les rotifères dominent en été et en automne dans les bassins primaires, suivis des protozoaires ciliés en hiver dans les bassins intermédiaires, et du phyllopode Artemia qui représente l'essentiel du zooplancton dans les bassins terminaux et atteint une abondance maximale au printemps. Les résultats de cette étude permettent de penser que la salinité, dont les effets sont accentués dans les zones arides, représente, probablement en conjonction avec les ressources, un paramètre déterminant et sélectif pour la diversité et la dynamique des peuplements planctoniques dans la saline de Sfax et les marais salants en général. 


\title{
Étude qualitative et quantitative des peuplements phyto- et zooplanctoniques dans les bassins de la saline de Sfax, Tunisie
}

\author{
Qualitative and quantitative study of phyto- \\ and zooplankton communities in the saline pounds \\ of Sfax, Tunisia
}

H. AYADI ${ }^{1}$, N. TOUMI ${ }^{1}$, O. ABID ${ }^{1}$, K. MEDHIOUB $^{2}$, M. HAMMAMI $^{3}$, T. SIME- NGANDO ${ }^{*}$, C. AMBLARD ${ }^{4}$, D. SARGOS ${ }^{4}$

\section{SUMMARY}

Paralic ecosystems are transitional systems between marine and continental areas. Thalassohaline waters where brines are formed by evaporation of seawater in land-locked bays or lagoons, such as salt pans or salt works, are representative of these systems. Except for the studies on the ecology and biogeography of the brine shrimp Artemia, few attemps have been made to analyze phyto- and zooplankton communities in salt works, especially those located in zones subject to arid conditions. Herein, we examined the seasonal dynamics of phyto- and zooplankton assemblages in a salt works located in the central area of the eastern coast of Tunisia ( $\mathrm{Sfax}, \sim 36^{\circ} \mathrm{N}, 11^{\circ} \mathrm{E}$ ), from August 1999 to May 2000 , concurrently with a variety of environmental parameters. Phyto- and zooplankton communities were substantially diversified and their dynamics were apparently in relation with the salinity in the pans and the resource availability. During the study, phytoplankton populations were quantitatively and successively dominated along the salt works by diatoms in primary pans (salinity $<200 \%$ ), followed by dinoflagellates in intermediate pans (salinity $=\mathbf{2 0 0 - 3 0 0} \%$ ), and by a near monospecific development of the chlorophycean Dunaliella salina in salt production pans. Zooplankton ( $>20 \mu \mathrm{m}$ ) organisms were quantitatively dominated by rotifers and/or copepods in primary pans in Summer and Autumn, by ciliated protozoa during Winter in intermediate pans, and by a near monospecific development of the brine shrimp Artemia in the salt production units of the salt works. Artemia exhibited their highest abundances in Spring. Overall, we

1. Laboratoire d'écobiologie animale, Département de biologie, Faculté des sciences, Université de Sfax, 3038 Sfax, Tunisie

2. Laboratoire de recherche des sciences de l'environnement (LARSEN), École nationale des ingénieurs, 3038 Sfax, Tunisie

3. Laboratoire de biochimie, Faculté de médecine, Monastir, Tunisie

4. Laboratoire de biologie des protistes, UMR CNRS 6023, Université Blaise Pascal (Clermont-Ferrand il), 63177 Aubière cedex, France

* Correspondance. E-mail : Telesphore.SIME-NGANDO@ibp.univ-bpclermont.fr

Les commentaires seront reçus jusqu'au 31 mars 2003. 
conclude that the diversity, the development and the dynamics of biological populations in the Sfax salt works are a consequence of the salinity of the milieu, likely coupled with the resource availability.

Key-words: salt pans, salt works, phytoplankton, zooplankton, population dynamics.

\section{RÉSUMÉ}

Le plancton végétal et animal a été analysé dans différents bassins de la saline de Sfax (Tunisie), entre Ie mois d'août 1999 et le mois de mai 2000. Des communautés diversifiées de phyto- et de zooplancton ont été observées. Quelle que soit la saison considérée, la structure des peuplements phytoplanctoniques se caractérise par la dominance de diatomées dans les bassins primaires de la saline (salinité $<200 \%$ ), suivie d'une dominance de dinoflagellés dans les bassins intermédiaires (salinité 200-300\%o), et d'un développement quasi-monospécifique de la chlorophycée Dunaliella salina dans les bassins terminaux où cristallise et se dépose la production saline (salinité $>300 \%$ ). En ce qui concerne le zooplancton $(>20 \mu \mathrm{m}$ ), les crustacés (copépodes) et les rotifères dominent en été et en automne dans les bassins primaires, suivis des protozoaires ciliés en hiver dans les bassins intermédiaires, et du phyllopode Artemia qui représente l'essentiel du zooplancton dans les bassins terminaux et atteint une abondance maximale au printemps. Les résultats de cette étude permettent de penser que la salinité, dont les effets sont accentués dans les zones arides, représente, probablement en conjonction avec les ressources, un paramètre déterminant et sélectif pour la diversité et la dynamique des peuplements planctoniques dans la saline de Sfax et les marais salants en général.

Mots clés : salines, marais-salants, phytoplancton, zooplancton, dynamique des peuplements.

\section{1 - INTRODUCTION}

Les milieux paraliques représentent, par leur situation géographique, des systèmes de transition entre le domaine marin et le domaine continental, dont la génèse se fait par restriction physiographique limitant les échanges avec la mer (KIENER, 1978). Les marais salants constituent un exemple de tels écosystèmes (DAVIS, 1977 ; 1978). Parmi les facteurs du milieu régissant le développement des communautés biologiques dans les marais salants, la morphométrie des bassins, l'hydrologie et la salinité jouent un rôle prépondérant (HEDGPETH, 1959 ; DAVIS, 1978). II en est de même du climat, notamment dans les zones arides où le bilan hydrique annuel peut être déficitaire, situation pouvant engendrer l'installation de conditions de sursalure dans les marais, qui deviennent alors des milieux extrêmes (TRABELSI, 1986 ; AMDOUNI, 1987 ; TOUMI, 1998).

En dehors des études concernant l'écologie et la biogéographie de la crevette d'intérêt économique Artemia (PERSOONE et SORGELOOS, 1980 ; KIENER, 1978), très peu d'études se sont penchées sur la composition et la dynamique des peuplements biologiques dans les milieux paraliques (DAVIS 1977, 1978 ; NOEL, 1982 ; THOMAS et GEISLER, 1982). À notre connaissance, aucune étude 
n'a été entreprise dans des marais salants situés en zone aride. La connaissance des peuplements planctoniques et des modalités de transfert de matière et d'énergie dans les réseaux trophiques des marais salants constitue donc l'objet principal de la présente étude.

II s'agit, plus précisément, d'étudier la composition spécifique et les variations spatiotemporelles des communautés phyto- et zooplanctoniques (protoet métazooplancton) dans les bassins de la saline de Sfax (Tunisie), en relation avec des paramètres physicochimiques du milieu. En raison de la situation de cette saline en zone aride, nous émettons l'hypothèse selon laquelle les variations de la salinité, accentuées par un bilan hydrique négatif, représentent, en conjonction avec les ressources trophiques disponibles, un paramètre déterminant pour la diversité, le développement et la dynamique des peuplements planctoniques.

\section{2 - MATÉRIEL ET MÉTHODES}

\subsection{Caractéristiques du site d'étude}

La saline de Sfax a été construite dans la zone centrale de la côte Est de la Tunisie, à environ $36^{\circ} \mathrm{N}$ et $11^{\circ} \mathrm{E}$, au Sud de la ville de Sfax (figure 1). Cette localité est caractérisée par un climat aride, le bilan hydrique étant négatif et donc déficitaire (figure 2), ce qui contribue aux fortes valeurs de salinité, dès les bassins de tête de la saline (TRABELSI, 1986 ; AMDOUNI, 1987 ; TOUMI, 1998). Cet écosystème présente une hydrologie artificielle, dans la mesure où la prise d'eau et la circulation de cette eau dans les différents bassins de la saline sont entièrement contrôlées. La saline s'étend sur une superficie totale de 1500 ha, la superficie évaporatoire étant de l'ordre de 1350 ha, dont environ $10 \%$ est réservée aux tables salantes (figure 1). La période d'activité de la saline est fixée par les conditions météorologiques, la production annuelle oscillant autour de 300000 tonnes de halites et 25000 tonnes de saumures magnésiennes.

L'alimentation de la saline à partir des eaux de la Méditerranée se fait à marée haute. Les eaux entrant dans la saline passent successivement dans 6 types de bassins qui diffèrent par leur concentration en sel : les bassins dits d'avants-pièces (superficie $=640$ ha, salinité des eaux $=75 \%$ ), les " partènements " extérieurs (450 ha, $130 \%$ ), les " partènements » intérieurs (160 ha, $210 \%$ ), les avants-pièces maîtresses (60 ha, $270 \%$ ), les pièces maîtresses (20 ha, $300 \%$ ) et, enfin, les tables salantes (14 ha) qui sont, en réalité, les bassins de dépôt de halite.

\section{2 Échantillonnage}

Les échantillons ont été prélevés toutes les trois semaines entre le mois d'août 1999 et le mois de mai 2000, à l'aide d'une bouteille fermante de type Van Dorn. Suite aux résultats d'une étude préliminaire (TOUMI, 1998), 7 ou 8 stations différentes ont été échantillonnées, en tenant compte des caractéris- 


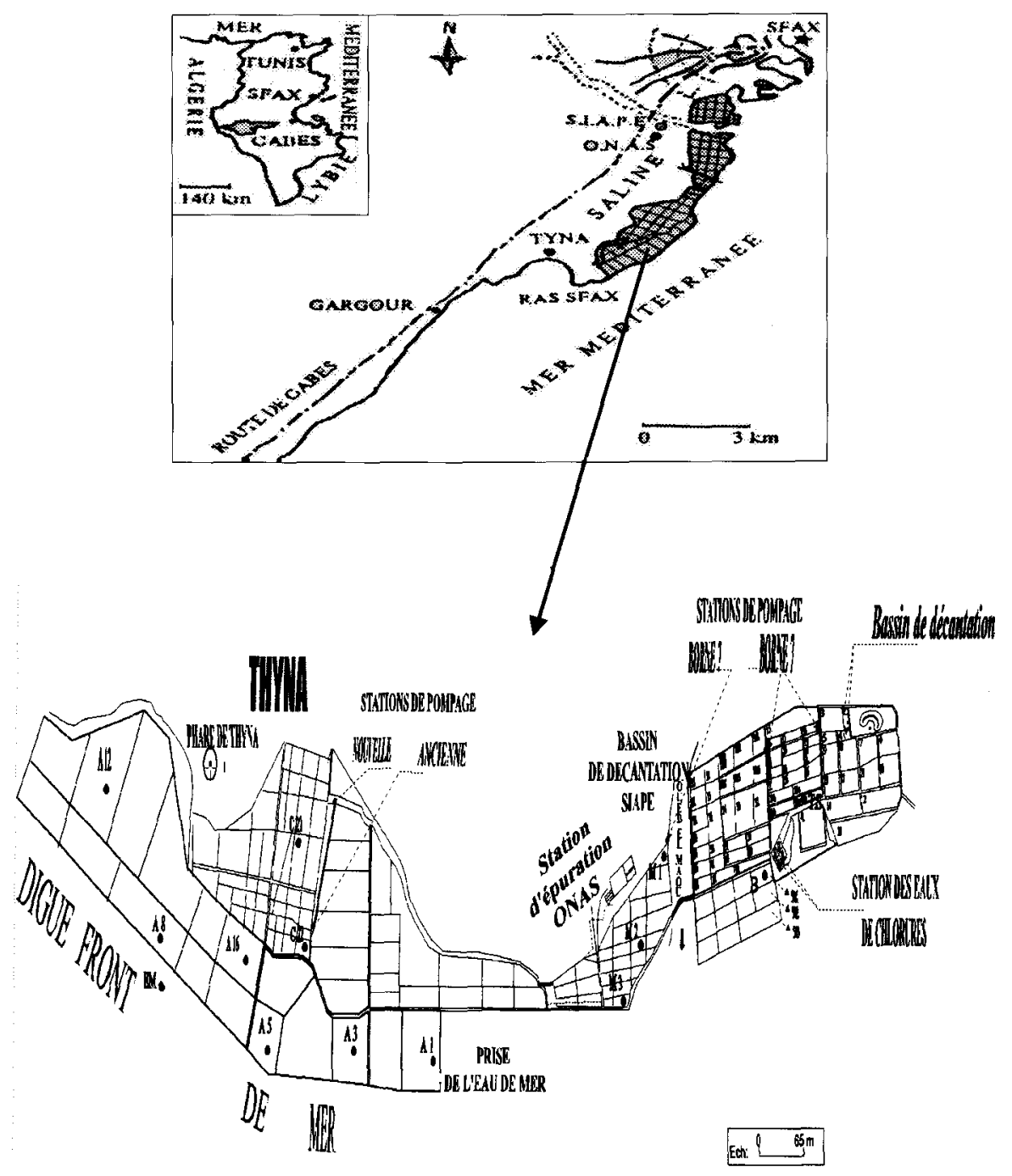

Figure 1 Localisation de la saline de Sfax (Tunisie) et des stations d'étude. Location of the salt works of Sfax (Tunisia) and the sampling stations.

tiques physicochimiques de l'eau, de la surface des bassins et du gradient de salinité le long de la chaîne de concentration des halites. Les différentes stations d'échantillonnage sont les suivantes : une station d'eau de mer (EM), trois stations dans les avants-pièces de la saline ( $A 1, A 5$ et $A 16)$, une station dans les « partènements " extérieurs (C2-1), une ou deux stations dans les pièces maîtresses ( $\mathrm{M} 1$ pour le zooplancton uniquement, M2 pour tous les autres paramètres, y compris le zooplancton) et, enfin, une station située au niveau des tables salantes (TS) (figure 1). 


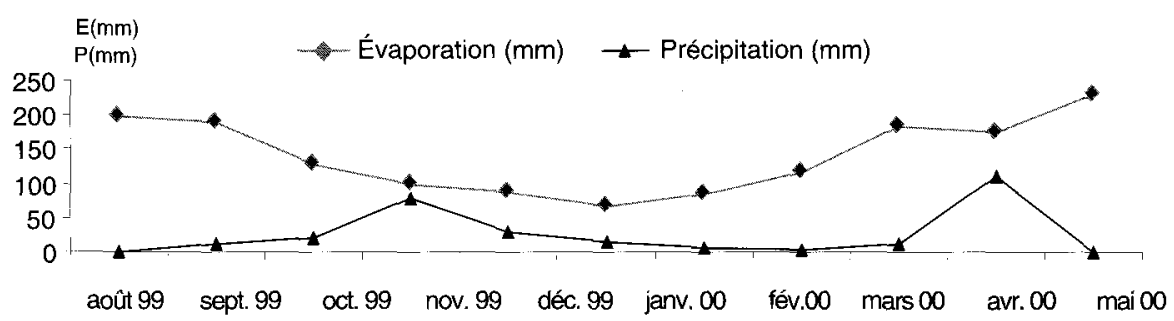

Figure 2 Variations des précipitations et de l'évaporation dans la région de Sfax au cours de la période d'étude.

Variations of precipitations and of evaporation in the region of Sfax during the study.

\subsection{Méthodes analytiques}

La température $\left({ }^{\circ} \mathrm{C}\right)$ a été mesurée in situ à l'aide d'un thermomètre classique incorporé à la bouteille Van Dorn, et la salinité (\%o) grâce à la méthode du résidu sec. L'acidité ionique (unités $\mathrm{pH}$ ) dans les bassins a été déterminée à l'aide d'un $\mathrm{pH}$-mètre de type Tacussel. Les concentrations en MES $\left(\mathrm{g} \cdot \mathrm{L}^{-1}\right)$ correspondent au poids sec, par unité de volume, de matières en suspension préalablement retenues par filtration sur des membranes de $0,45 \mu \mathrm{m}$ de porosité. Pour l'ensemble des paramètres physicochimiques, seuls les résultats obtenus à l'entrée (i.e. eau de mer, station EM) et à la sortie (tables salantes, station TS) de la saline sont présentés dans le cadre de la présente étude.

Les échantillons (200 $\mathrm{mL}$ ) destinés à l'analyse du phytoplancton et des protozoaires ciliés ont été fixés au Lugol (conc. finale $1 \%$ vol./vol.), les dénombrements étant effectués en microscopie inversée d'après la méthode de UTHERMÖHL (1958). La systématique des espèces phytoplanctoniques a été réalisée en se référant aux ouvrages de BOURRELLY (1966) et de GERMAIN (1981). Pour le zooplancton métazoaire, les organismes contenus dans 10 litres d'échantillon sont concentrés à l'aide d'un filet à zooplancton de type Juday de $70 \mu \mathrm{m}$ de vide de maille, fixés au formaldéhyde (conc. finale $4 \%$ ) et dénombrés sous une loupe binoculaire.

\section{3 - RÉSULTATS ET DISCUSSION}

\subsection{Environnement physicochimique}

Les variations temporelles des paramètres physicochimiques mesurés au cours de cette étude dans les stations EM et TS sont présentées sur la figure 3. La température dans les différents bassins de la saline a fluctué de $10^{\circ} \mathrm{C}$ en hiver à $35^{\circ} \mathrm{C}$ en été, les variations saisonnières marquées de ce paramètre étant caractéristiques des zones méditerranéennes. La salinité de l'eau passe d'environ $45 \%$ dans les bassins de tête de la saline recevant les eaux marines 

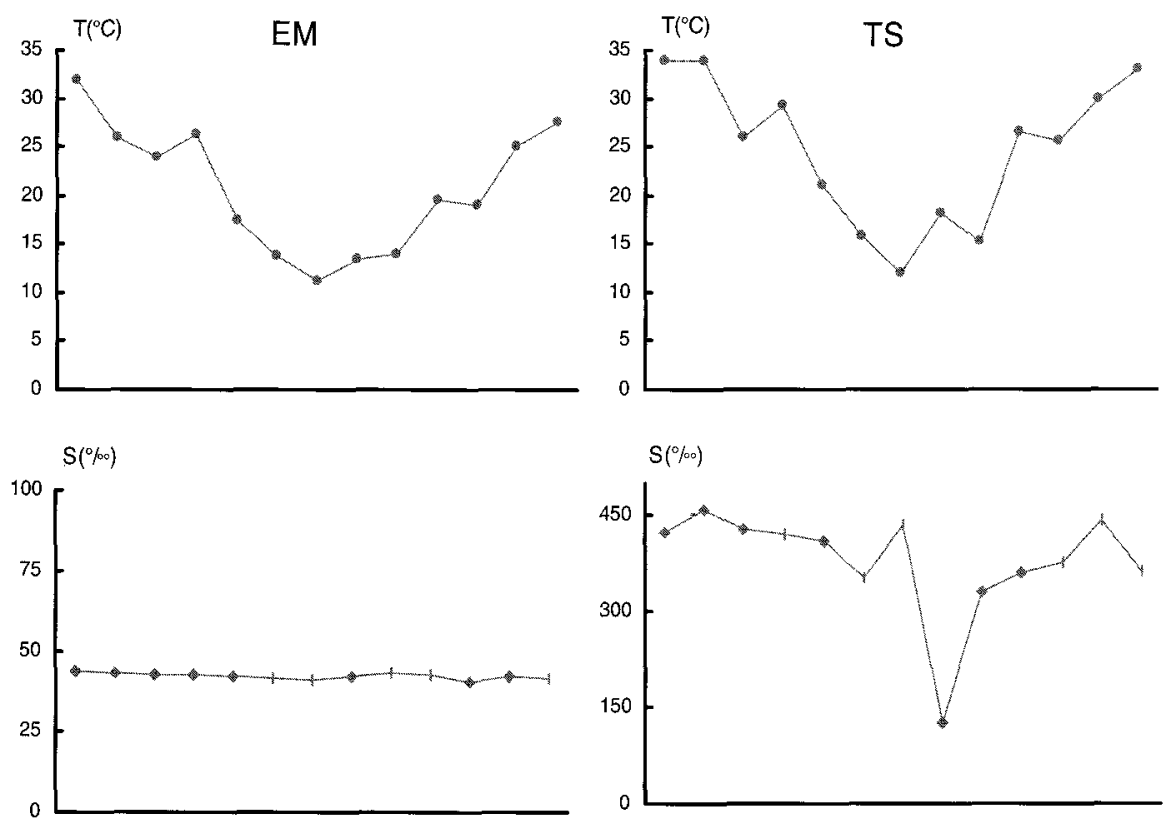

$\mathrm{pH}$

$\mathrm{pH}$
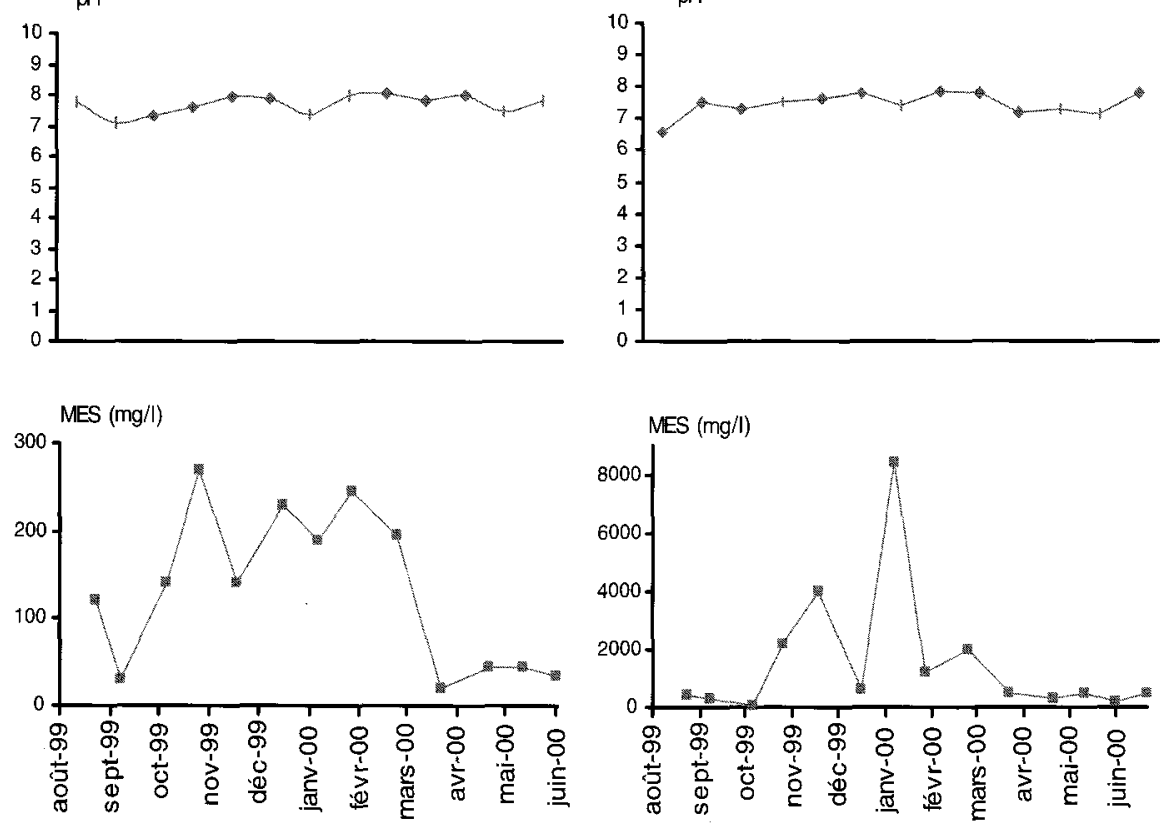

Figure 3 Variations des paramètres physicochimiques pris en compte au cours de l'étude dans les stations EM (eau de mer) et TS (tables salantes).

Variation of the physico-chemical parameters taken into account during the study at stations EM (marine waters) and TS (salt production pounds). 
(station EM), à des valeurs 10 fois plus élevées au niveau des tables salantes (station TS). Les fluctuations temporelles de l'acidité ionique dans les bassins sont relativement faibles, les valeurs de $\mathrm{pH}$ étant globalement comprises entre 7 et 8 . L'apparition ponctuelle de $\mathrm{pH}$ légèrement acides dans les bassins est, sans doute, liée à l'activité de bactéries sulfatoréductrices (AMIT et BENTOR, 1971).

La turbidité des eaux augmente avec la salinité des bassins, les eaux étant nettement plus claires à l'entrée de la saline (station EM) où les teneurs en MES sont $<300 \mathrm{mg} \cdot \mathrm{L}^{-1}$. Au niveau des tables salantes, des concentrations en MES très largement supérieures à $1000 \mathrm{mg} \cdot \mathrm{L}^{-1}$ ont été enregistrées en plusieurs occasions. La forte turbidité dans les bassins les plus salés est liée, non seulement à la cristallisation (AMDOUNI, 1987), mais également aux facteurs biotiques tels que le développement phytoplanctonique et la dégradation du matériel biologique provenant des avants-pièces de la saline (TRABELSI, 1986 ; TOUMI, 1998). Des observations similaires ont été faites dans les marais salants de Giraud (France), où la forte turbidité et la couleur des eaux dans les bassins les plus salés ont été mises en relation avec un important développement bactérien (CLASTERE, 1984). D'un point de vue temporel, les concentrations en MES sont plus élevées en automne et en hiver qu'au printemps et en été. Cela est naturellement lié au fort ensoleillement local au printemps et en été, qui provoque une photo-inhibition pour l'activité biologique et, notamment, pour le développement du phytoplancton (TOUMI, 1998 ; TRABELSI, 1986).

\subsection{Peuplements phytoplanctoniques}

Malgré la prévalence de conditions contraignantes voire extrêmes dues à la salinité, une communauté phytoplanctonique abondante et diversifiée a été observée au cours de cette étude. En effet, plus de 80 espèces phytoplanctoniques appartenant à 5 groupes taxonomiques différents (diatomées, dinoflagellés, cyanobactéries, euglénophycées et chlorophycées), ont été identifiées (tableau 1). Cependant, l'abondance phytoplanctonique est largement dominée, au cours de cette étude, par des diatomées et des dinoflagellés dans les eaux marines arrivant dans la saline et dans les bassins formant les avants-pièces et les « partènements " extérieurs, alors que les chlorophycées forment l'essentiel du peuplement phytoplanctonique dans les pièces maîtresses et les tables salantes. L'importance relative moyenne des principaux groupes taxonomiques dans les différents bassins échantillonnés est présentée sur la figure 4.

Dans les stations EM et $A 1$, les diatomées forment l'essentiel du peuplement phytoplanctonique, puisqu'elles représentent, respectivement, 82 et $86 \%$ de l'abondance totale. Les dinoflagellés représentent $11 \%$ de l'abondance phytoplanctonique totale dans ces deux stations, formant ainsi le deuxième groupe phytoplanctonique d'un point de vue quantitatif. Dans les bassins formant les avants-pièces de la saline (stations A5 et A16) et dans les «partènements » extérieurs (station C2-1), les dinoflagellés se développent intensément pour former, respectivement, 80,77 et $68 \%$ de l'abondance phytoplanctonique totale, au détriment des diatomées qui ne représentent plus que 19, 23 et $32 \%$ de l'abondance totale. Dans les pièces maîtresses (station M2), la chlorophycée Dunaliella salina (66\% de l'abondance totale) se développe préférentiellement, accompagnée des dinoflagellés (25\%) et des diatomées (7\%). Au niveau des tables salantes (station TS), on assiste à une 
Tableau 1 Liste qualitative des espèces phytoplanctoniques identifiées dans la saline de Sfax d'août 1999 à juin 2000.

Table 1 Qualitative list of the phytoplancton species identified in the Sfax salt works from August 1999 to June 2000.

\section{Cyanobactéries}

Oscillatoria sp.

Sirulina subsalsa

\section{Diatomées}

pennées

Amphiprora alata

Asterionella sp.

Climacosphenia sp.

Diatomella sp.

Donkinia lata

Epithemia turgida

Gyrosigma balticum

Gyrosigma sp.

Navicula sp.1

Navicula sp. 2

Navicula sp. 3

Nitzschia sp.

Nitzschia acicularis

Nitzschia insignes

Nitzschia longissuma

Nitzschia valida

Nitzschia ventricosa

Pinnularia sp.

Pleurosigma sp.

Pleurosigma ituim

Pleurosigma sp.1

Pleurosigma sp.2

Stenopterobia intermedia

Surirella sp.

\section{centriques}

Biddulphia mobiliensis

Biddulphia sp.1

Biddulphia sp.2

Chaetoceros $\mathrm{sp}$.

Chaetoceros contrictus

Chaetoceros didymus

Chaetoceros borenzianus

Chaetoceros similis

Coscinodiscus sp.

Coscinodiscus stellaris

Rhizosolenia sp.

Rhizosolenia hebatata

Rhizosolenia setigera

Rhizosolenia styliformis

Skeletonema costatum

Thalassiosira mendiolana

Thalassiosira sp.

\section{Dinoflagellés}

Dubosquella tintinnicola

Glenodinium monensis

Gonyaulax sp.

Gymnodinium sp.

Gymnodinium sp.1

Gymnodinium sp.2

Gymnodinium sp.3

Gymnodinium caltenatum

Gymnodinium pulchellum

Gymnodinium simplex

Gymnodinium splendens

Gymnodinium veneficum

Gymnodinium vitiligo

Gyrodinium lachryma

Gyrodinium sp.

Heterocapsa sp.

Oxyrrhis marina

Peridinium sp.

Peridinium incospicuum

Peridinium platinium

Peridiniopsis sp.

Prorocentrum lima

Prorocentrum micans

Prorocentrum reticulatum

Prorocentrum sp.

Protogonyaulax sp.

Protoperidinium sp.

Protoperidinium conicum

Protoperidinium minutum

Protoperidinium ovatum

Protoperidinium pellucidum

Protoperidinium sp.

Protoperidinium sp.1

Scripsiella trochoïdea

Sphaerodinium polonicum

\section{Chlorophycées}

Dictyocha fibula

Dunaliella salina

Chlamydomonas sp.

Phyllariochloris sp.

Phyllariochloris coeca

\section{Euglenophycées}

Euglena sp. 
EM

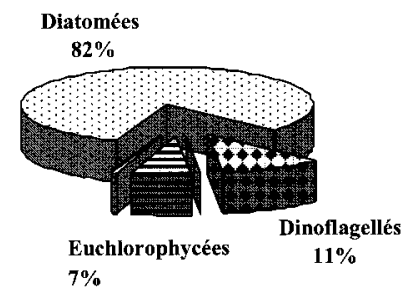

A5

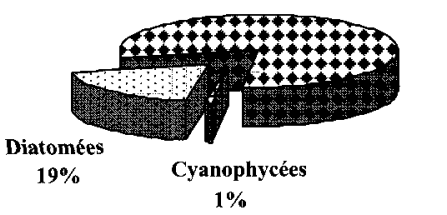

C2-1

Dinoflagellés $68 \%$

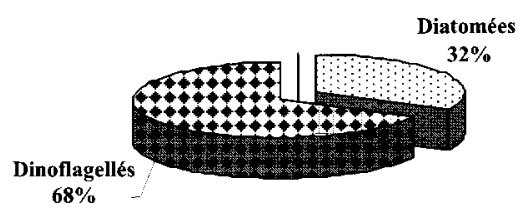

$\mathbf{A 1}$

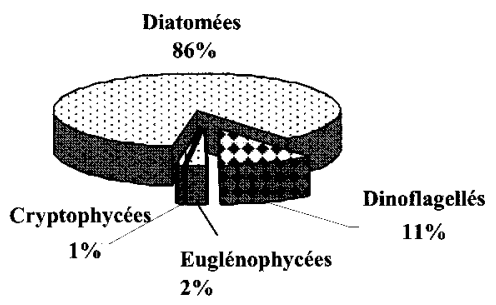

A16

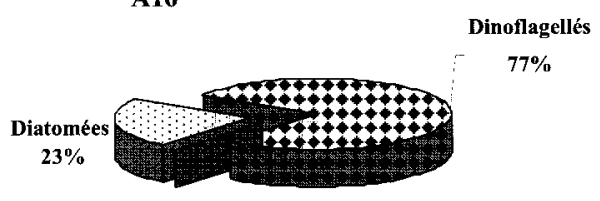

M2

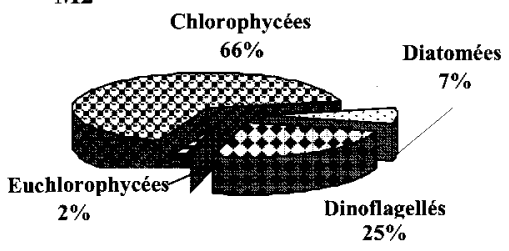

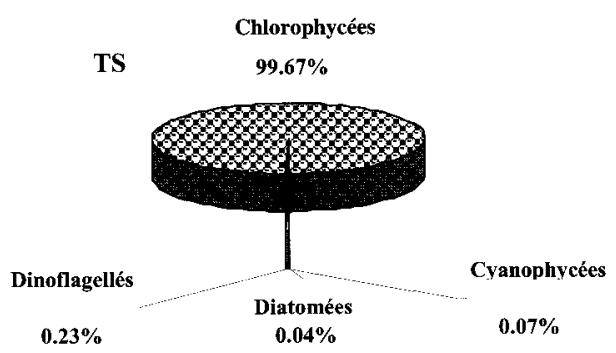

Figure 4 Importance relative de l'abondance des différents groupes phytoplanctoniques observés au cours de l'étude, dans les stations échantillonnées de la saline de Sfax.

Relative importance of the abundance of the different phytoplanktonic groups observed during the study in the sampled stations in the Sfax salt works.

floraison quasi-monospécifique de $D$. salina ( $>99 \%$ de l'abondance totale). Cette floraison est à l'origine de la couleur " lie de vin " des eaux dans les bassins formant les pièces maîtresses et les tables salantes.

La dynamique des populations phytoplanctoniques présentes dans la saline de Sfax est donc dépendante de la salinité. L'effet sélectif de ce paramètre contraignant réduit, pour les plus fortes concentrations, considérablement la 
richesse et la diversité spécifiques, aboutissant au niveau des tables salantes à une communauté algale à tendance monospécifique très marquée, où domine largement l'espèce $D$. salina. Ce phénomène a également été observé dans différents milieux similaires, notamment dans les marais salants de Giraud en France (THOMAS et GEISLER, 1982 ; NOEL, 1982) et de Long Island aux ÉtatsUnis d'Amérique (DAVIS, 1978), de même que dans la lagune de Boughrera en Tunisie (KEFI, 1993).

\subsection{Peuplements zooplanctoniques}

Les variations saisonnières de l'abondance du zooplancton total et des groupes dominants, à savoir les copépodes, les rotifères et les protozoaires ciliés, dans les différents types de bassins de la saline de Sfax, sont représentées sur la figure 5. De manière générale, les densités les plus élevées du zooplancton sont enregistrées dans les bassins formant les avants-pièces et/ou les "partènements " extérieurs de la saline en été et en automne, alors qu'en hiver et au printemps, les plus fortes abondances du zooplancton sont plutôt observées dans les bassins formant les pièces maîtresses de la saline. Cette distribution spatiotemporelle est étroitement liée au fait que : (i) le zooplancton crustacé (copépodes) et les rotifères se développent essentiellement en été et en automne et presque uniquement dans les eaux marines (station EM), les avants-pièces (stations A1, A5 et A16) et les " partènements " extérieurs (station C2-1) de la saline, (ii) les protozoaires ciliés, organismes unicellulaires de plus petite taille, se développent préférentiellement dans les pièces maîtresses de la saline (stations M1 et M2) où ils atteignent une abondance maximale en hiver, et (iii) au printemps, les ciliés sont remplacés par le phyllopode Artemia qui forme l'essentiel du peuplement zooplanctonique dans les stations M2 et TS (figure 5). L'augmentation considérable de la salinité le long de la chaîne de concentration des halites représente donc un facteur essentiel pour la structure et la dynamique du zooplancton dans la saline de Sfax. Tout comme pour le phytoplancton, cette augmentation de salinité, accentuée par un bilan hydrique déficitaire (figure 2), réduit considérablement la richesse et la diversité spécifiques du zooplancton qui, au niveau des tables salantes, est presque exclusivement représenté par le phyllopode Artemia.

II est important de noter que le développement important de rotifères observé aux stations A16 et C2-1 en août et en novembre, fait suite à un bloom phytoplanctonique. Par ailleurs, les abondances les plus élevées des organismes de petite taille, notamment les protozoaires ciliés, ont été observées dans les pièces maîtresses de la saline et en hiver (figure 5). II en est de même pour les stades larvaires et les nauplii de copépodes. II est admis que les organismes de petite taille s'adaptent mieux aux conditions contraignantes du milieu, en raison, notamment, d'un taux de renouvellement élevé assimilable à une stratégie démographique de type $r$, qui leur permet de répondre rapidement aux conditions fluctuantes du milieu (AMBLARD et al., 1998). Enfin, l'ensemble des résultats de cette étude nous amène à considérer que la salinité représente, sans doute en conjonction avec les ressources, un facteur de contrôle important pour la dynamique des peuplements planctoniques de la saline de Sfax et des marais salants. 

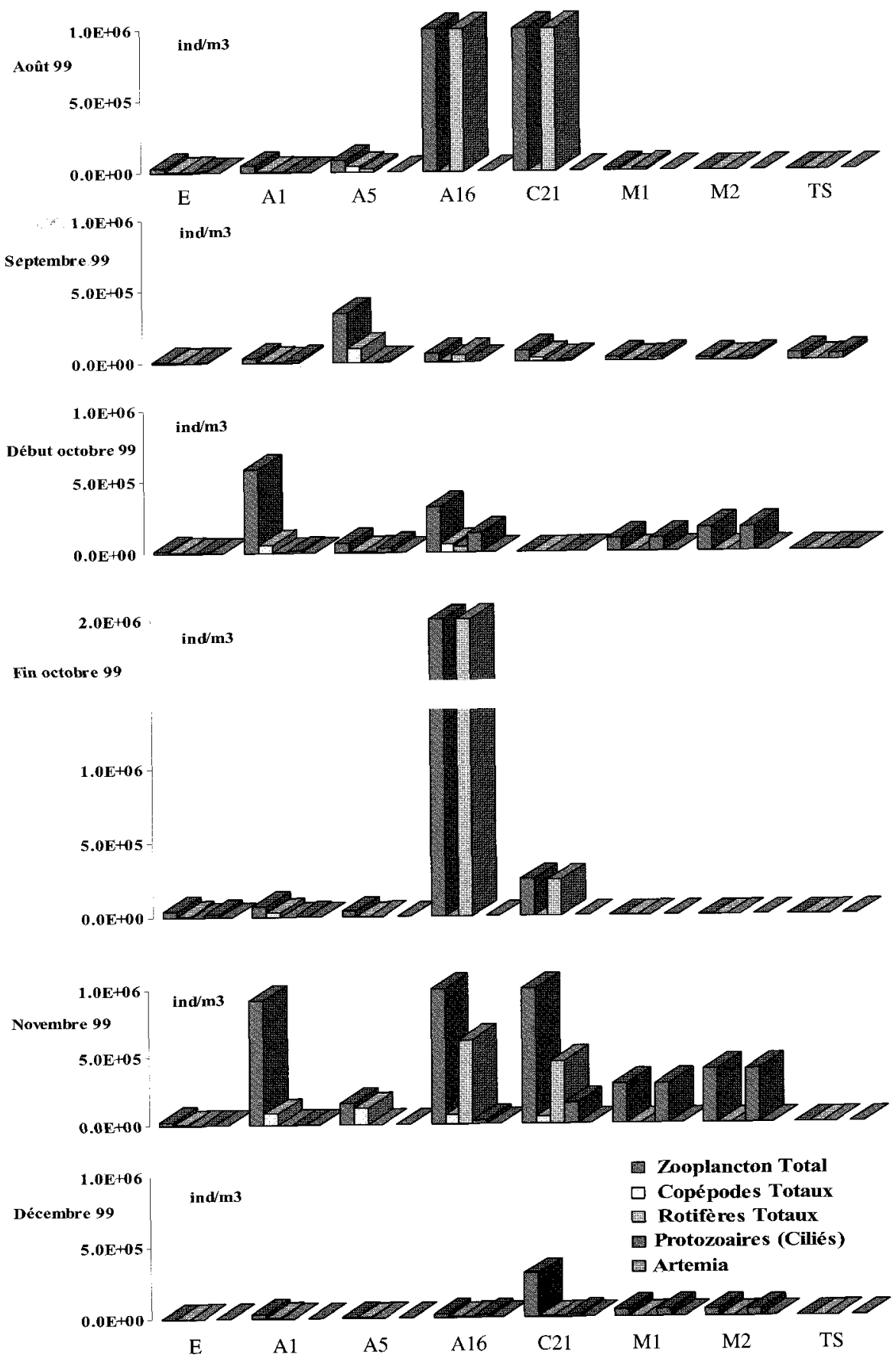

Figure 5 Variations spatiotemporelles de l'abondance du zooplancton dans la saline de Sfax.

Spatial and temporal fluctuations of the abundance of zooplankton in the Sfax Salina. 


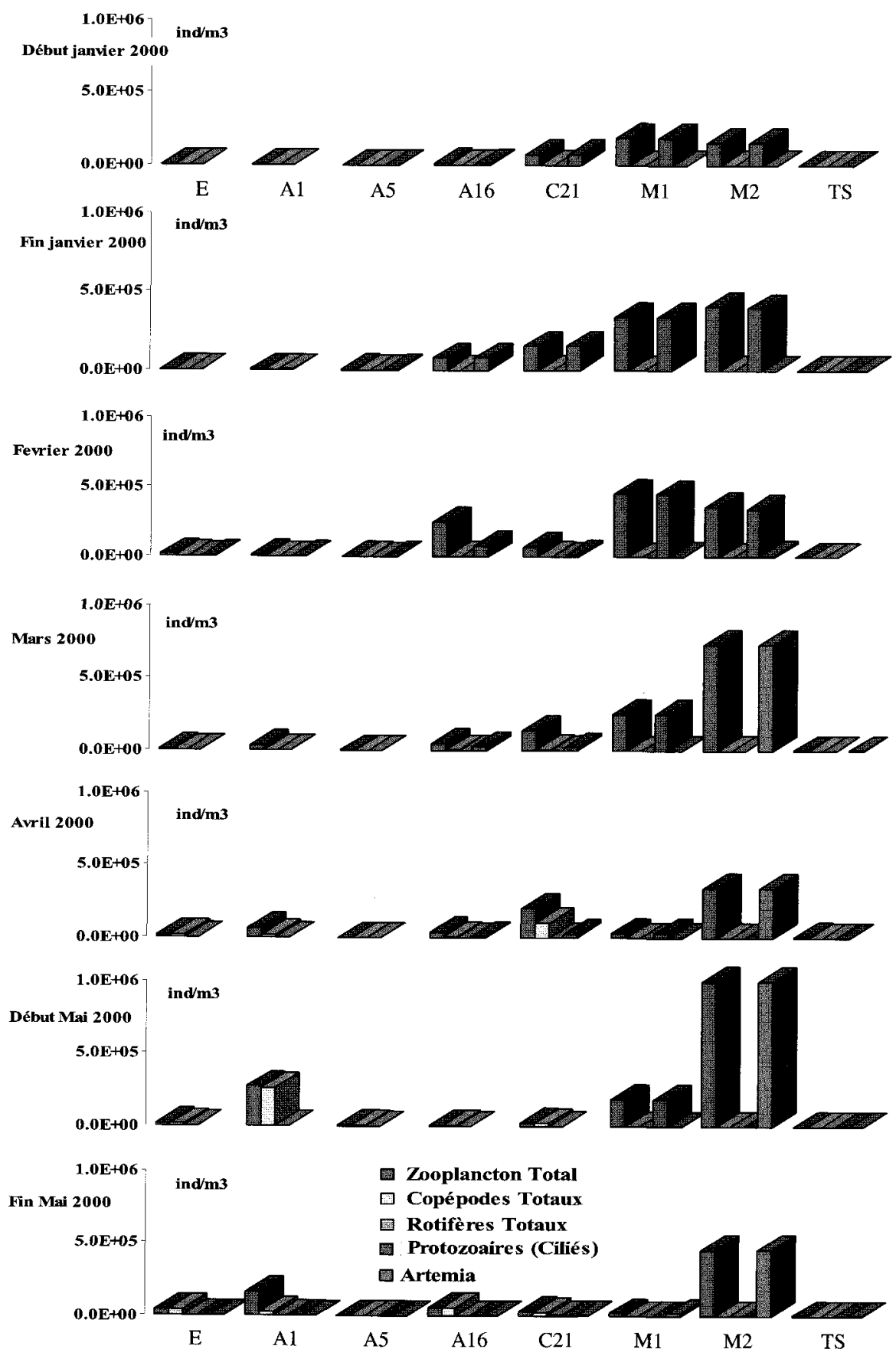

Figure 5 (continued) Variations spatiotemporelles de l'abondance du zooplancton dans la saline de Sfax.

Spatial and temporal fluctuations of the abundance of zooplankton in the Sfax Salina. 


\section{RÉFÉRENCES BIBLIOGRAPHIQUES}

AMBLARD C., BOISSON J.C., BOURDIER G., FONTVIEILLE D., GAYTE X., SIMENGANDO T., 1998. Écologie microbienne en milieu aquatique : des virus aux protozoaires. Rev. Sc. Eau, n spécial, 145162.

AMDOUNI R., 1987. Étude géochimique des saumures libres, des sédiments et des sels dans la saline de Sfax (Tunisie). Th. Doct. Univ. Paris VII, 249 p.

AMIT O., BENTOR Y.K., 1971. pH-dilution curves of saline waters. Chem. Geol., 7, 307-813.

BOURRELLY P., 1966. Les algues d'eau douce; Boubée, Paris.

CLASTERE J., 1984. De la mer aux évaporites : biomasse et production du maillon primaire d'un marais salant, Camargue. Th. Doct. Univ. Pierre \& Marie Curie, Paris VI, $421 \mathrm{p}$.

DAVIS J.S., 1977. Importance of micro-organismes in solar salt production. In: "4 th Symposium on salt", COOGAN A.H. [ed.], 369-372.

DAVIS J.S., 1978. Biological communities in ambient enriched salina. Aquatic Botany, 4, 29-42.

GERMAIN H., 1981. Flore des diatomées des eaux douces et saumâtres, Boubée, Paris.

HEDGPETH J.W., 1959. Some preliminary considerations of the biology of inland mineral waters. Archivio Oceanogr. Limnol., 11, 111-141.
KEFI O., 1993. Contribution à l'étude du milieu et du phytoplancton de la lagune de Boughrera. DEA, Univ. du Sud, Sfax, Tunisie, $197 \mathrm{p}$.

KIENER A., 1978. Écologie, physiologie et économie des eaux saumâtres, Masson, Paris.

NOEL D., 1982. Les diatomées des saumures des marais salants de Giraud (Sud France). Geol. Medit., 4, 413-446.

PERSOONE G., SORGELOOS P., 1980. General aspects of the ecology and biogeography of Artemia. In: PERSOONE G., SORGELOOS P., ROELS O., JASPER E. (ed.), The brine shrimp Artemia, Vol. 3 : ecology, culturing, use in aquaculture, 324.

THOMAS J.S., GEISLER D., 1982. Peuplements benthiques à cyanophycées des marais salants de Giraud (Sud France). Geol. Medit., 4, 391-411.

TOUMI N., 1998. Contribution à l'étude géochimique et biologique des marais salants de la saline de Sfax. DEA, Univ. du Sud, Sfax, Tunisie, $102 \mathrm{p}$.

TRABELSI W., 1986. Étude géochimique et microbiologique des saumures de la saline de Sfax. DEA, Univ. du Sud, Sfax, Tunisie, $60 \mathrm{p}$.

UTHERMÖL H., 1958. Zur Vervollkommnung der quantitativen Phytoplankton-Methodik. Mitt. Int. Verein. Limnol, 9, 1-38. 